\title{
VIOLENCIA DOMÉSTICA: \\ UN MODELO INTEGRAL DE INTERVENCIÓN ${ }^{1}$
}

\section{María M. Lizardi López ${ }^{2}$}

\section{Resumen}

El artículo presenta algunas reflexiones sobre un modelo integral de intervención con sobrevivientes de violencia doméstica y con agresores desarrollado bajo los auspicios del Programa de Apoyo Familiar a Niños y Adultos (A.F.A.N.A.) del Intitute for Individual, Group, and Organizational Development, Inc. una organización sin fines de lucro en Gurabo, Puerto Rico. El modelo presentado tiene un enfoque de prevención a los diferentes niveles con una fuerte influencia de las perspectivas eco-sistémicas, de apoderamiento y fortalezas, de la perspectiva de resiliencia y del construccionismo social. El artículo presenta alternativas de prevención e intervención a nivel primario y secundario con la violencia doméstica. La autora presenta recomendaciones para abordar el problema de la violencia doméstica desde diferentes frentes y con diferentes estrategias. Dichas recomendaciones conllevan la activación de todos los sectores sociales, el enfoque de prevención y la revisión de la política social. La autora invita a formar una voluntad colectiva que nos lleve a un compromiso hacia la eliminación o disminución al máximo de la violencia doméstica.

Descriptores: modelo integral de intervención, sobrevivientes, violencia doméstica, agresores, perspectiva eco-sistémica, apoderamiento y fortalezas, resiliencia, construccionismo social.

\footnotetext{
'Artículo basado en la ponencia presentada en la actividad educativa: Violencia doméstica: Desafortunadamente tenemos que seguir hablando. Universidad Interamericana de Puerto Rico, el 3 de noviembre de 2006.

${ }^{2}$ Catedrática de la Escuela de Trabajo Social, Programa Graduado de la Universidad Interamericana de Puerto Rico, Recinto Metropolitano.
} 


\section{Abstract}

The article presents an integrated model for the intervention with survivors and aggressors of domestic violence developed under the auspices of the Intitute for Individual, Group, and Organizational Development, Inc., a non for profit organization in Gurabo, Puerto Rico. The model discussed has a preventive approach at different levels. It has a strong influence of ecosystemic, empowerment, strengths and resilience perspectives, and social constructionism. The paper presents alternatives for primary and secondary prevention of domestic violence. The author presents specific recommendations for the elimination or control of the problem of domestic violence including the diverse sectors of society and different strategies. The recommendations placed an important emphasis to the analysis and revision of social policy related to families and domestic violence. The author invites to create a collective effort towards the commitment necessary for the elimination or control of domestic violence.

Keywords: Integrated intervention model, domestic violence survivors, aggressors, eco-systemic, empowerment, strengths, resilience perspectives and social constructionism.

\section{Introducción}

El tema de la violencia doméstica es uno tratado con mucha frecuencia tanto en la literatura profesional como en los medios, a veces de forma dramática o de forma trivial. Si embargo es un problema que no deja de preocuparnos como trabajadores y trabajadoras sociales, ya que refleja que aún hay mucho por hacer para eliminar o disminuir dicho problema al máximo. Esto es de suma importancia si consideramos el alto número de hogares afectados por este problema en Puerto Rico y en otros países. Recuerdo con suma tristeza que el mismo día que presenté la ponencia que dio origen a este artículo un hombre asesinó a su esposa y luego se suicidó en la ciudad de Vega Alta, Puerto Rico. A los pocos días la primera plana de un periódico local reseñaba la tragedia familiar de una joven pareja con dos niños menores 
que quedaron huérfanos, ya que el padre de éstos asesinó a su cónyuge y luego se privó de la vida. El padre era un policía.

Toda esta situación ha llegado a un punto en que parece que es necesario que todos y todas reflexionemos en nuestro rol como partes de la solución del problema. Todo esto corrobora el hecho de que: "desafortunadamente tenemos que seguir hablando," como fue el título de la actividad educativa que dio origen al presente artículo y yo le añado: "y actuando a diferentes niveles, con diferentes estrategias y nuevos paradigmas".

No es el propósito del presente trabajo presentar estadísticas que hablen de la magnitud del problema, pues es altamente conocido y es muy fácil tener acceso dicha información a través del Internet y a otros recursos disponibles. Tampoco entraré en detalles descriptivos de definiciones de lo que es violencia doméstica, los tipos de maltrato de acuerdo a la ley, el ciclo de violencia, las teorías que tratan de explicar su origen, etc. Mi interés particular en el presente trabajo es compartir algunas reflexiones sobre un modelo integral de intervención con la violencia doméstica que he tenido la oportunidad de conceptuar y llevar a cabo con éxito en la práctica del trabajo social en Puerto Rico. Esto con el propósito de ser co-partícipe en el proceso de buscar alternativas hacia la eliminación de la violencia doméstica e invitar a otros profesionales a hacer lo mismo, considerando que no existe una sola solución para un problema tan antiguo y tan complejo.

El modelo de intervención descrito fue desarrollado bajo los auspicios del Programa de Apoyo Familiar a Niños y Adultos (A.F.A.N.A.) del Intitute for Individual, Group, and Organizational Development, Inc. Este instituto es una organización sin fines de lucro con sede en la ciudad de Gurabo, Puerto Rico. Su misión es contribuir a la transformación de estructuras e ideologías sociales para promover relaciones humanas de justicia social y de paz a través de diversas estrategias de prevención primaria y secundaria. 


\section{Base Teórica}

La base teórica de este modelo de intervención es ecléctica y toma algunas de las proposiciones de los siguientes marcos conceptuales:

- Perspectiva de apoderamiento (Fong, 2002; Gutiérrez, Parsons \& Cox, 2003). El modelo visualiza la capacidad de la persona de ganar control sobre su vida y de cambiar las condiciones que limitan su desarrollo y bienestar mediante esfuerzos individuales y colectivos. Por consecuencia se visualiza a las personas servidas como actores y actrices principales en la definición y manejo de sus situaciones de vida. El trabajador o trabajadora social sirve de intercesor o de intercesora de los y las participantes en situaciones que lo ameriten.

- Perspectiva de fortalezas (Saleebey, 1992; Poulin, 2005; Long, Tice \& Morrison, 2006). El modelo reconoce que toda persona, familia, grupo o comunidad posee fortalezas y hacia éstas se debe focalizar la intervención.

- Marco conceptual de prevención (Pransky, 1991; 2003). El modelo reconoce que existen factores que contribuyen al desarrollo de los problemas sociales y que los mismos pueden ser de carácter individual o estar enraizados en las estructuras sociales y las ideologías subyacentes de las mismas. A tono con eso la intervención no debe circunscribirse a una sola estrategia o nivel de intervención.

- Construccionismo social (Gergen, 1994; Gergen, MacNamee \& Barret,2001). El construccionismo sostiene que el mundo es socialmente construido, así como las diferentes realidades subjetivas que coexisten. Todo esto ocurre dentro de un contexto de una realidad social que incluye dimensiones económicas, políticas, sociales y culturales las cuales 
pueden apoyar o limitar nuestras narraciones y nuestra posibilidad de elección a determinados contextos (Bravo Urzúa, 2002).El construccionismo social presenta la importancia del lenguaje en la formación de la realidad subjetiva de cada persona y como un medio de diferenciación. Así cuando damos énfasis a ciertos aspectos de la realidad social como por ejemplo una actitud violenta estamos negando la realidad alterna que sería una actitud de paz. De esta manera la realidad social es construida día a día a través de nuestras comunicaciones e interacciones. Por lo tanto se puede afirmar que no hay una sola verdad objetiva y también que a cada realidad se antepone una realidad alterna (Gergen, 1994). El modelo propuesto reconoce la importancia de las relaciones sociales y la construcción y definición de aspectos como los roles de género y las relaciones familiares lo cual incide en el desarrollo de relaciones violentas en la pareja. Por ende, la intervención debe ir dirigida a examinar dichas construcciones sociales $\mathrm{y}$ a reconstruir junto a las personas, familias y grupos nuevas formulaciones que promuevan relaciones de justicia y paz.

- Perspectiva eco-sistémica (Germain y Gitterman,1991). El modelo visualiza a las personas en un proceso transaccional con el ambiente, en una relación recíproca y dinámica de influencia mutua. Las personas poseen capacidades de adaptación dinámica en las cuales pueden cambiarse a sí mismas, cambiar su ambiente o retirarse de ambientes nocivos. La violencia, el abuso de poder y la opresión en las relaciones sociales se visualizan como contaminación del ambiente social en las familias, grupos, comunidades y organizaciones. Se concibe la intervención a diferentes niveles (micro, mezzo y macro sistemas). 
- Modelo de intervención en crisis ( Slakieu, 1996; Molina 2000; James y Gilliland, 2005). El modelo reconoce que las personas, familias, grupos y comunidades pueden experimentar crisis. No obstante, las crisis pueden ser eventos que den lugar a un proceso de transformación y crecimiento. El trabajo social en situaciones de violencia doméstica debe visualizar las crisis como oportunidades de cambio y ayudar a los sistemas humanos con los que interviene a adquirir dicha visión.

- Perspectiva de resiliencia (Fraser, 2004; Greene \& Cohen, 2005; Walsh, 2006).El modelo reconoce que toda persona posee la capacidad de sobreponerse a circunstancias de gran adversidad y que dicha capacidad debe ser un eje central en la intervención en trabajo social.

El concepto de resiliencia es parte de los esfuerzos de los/as trabajadores sociales durante las últimas dos décadas como un nuevo paradigma para la práctica del trabajo social por lo cual deseo darle mayor énfasis en este escrito. Dicho concepto proviene de la palabra en latín resiliens que significa elasticidad y tiene varias definiciones, todas las cuales se refieren a la adaptación exitosa de una persona luego de haber experimentado un suceso de gran adversidad (Greene \& Cohen, 2005). Aún cuando se han llevado a cabo innumerables esfuerzos de trabajo científico sobre este concepto en diferentes disciplinas, no existe una teoría comprensiva sobre el mismo (Greene \& Conrad, 2002, pág.29). No obstante, existen algunos teóricos que consideran que la resiliencia como perspectiva teórica está lo suficientemente desarrollada como para servir de guía para la política y práctica de la salud mental (Begun, Fraser, Masten, Miller \& Macintosh como se cita en Greene y Conrad, 2002). 
Los postulados básicos expuestos por Greene (como se cita en Greene and Cohen, 2005) exponen que la resiliencia:

- es un fenómeno bio psicosocial y espiritual

- conlleva un proceso dinámico de intercambio personaambiente

- conlleva un proceso de adaptación

- ocurre en el transcurso de vida de individuos, familias y comunidades que experimentan desarrollos

- está ligado al estrés de vida y a las capacidades de enfrentamiento únicas de las personas

- conlleva competencia en el funcionamiento diario

- puede representar un continuo- un polo opuesto al riesgo

- puede ser interactivo, teniendo un efecto en combinación con factores de riesgo

- se amplía a través de la conexión o relación con otros

- esta influenciado por la diversidad lo cual incluye etnia, raza, género, edad, orientación sexual, status económico, afiliación religiosa y habilidad física y mental

Quisiera resaltar el hecho de que el aspecto de la espiritualidad es considerado en las perspectivas de fortalezas y de resiliencia. Greene y Conrad (2002) establecen la diferencia entre religión, fe y espiritualidad. La fe puede ser vista como el sistema de creencias acerca del sentido de la vida y la relación con lo trascendental o Dios. Por otro lado, la religión puede definirse como la expresión externa de la fe. La espiritualidad se refiere a la forma en que la persona "busca trascender el ser de manera que pueda descubrir significado, pertenencia y relación con lo infinito" (Conrad, citado en Greene \& Conrad, 2002, p.47). Mientras que la religión como expresión externa de la fe podría afectar positiva o negativamente el desarrollo del potencial humano, la espiritualidad se considera parte de los componentes de la resiliencia. En mi experiencia trabajando con pacientes de cáncer he encontrado que la espiritualidad ha sido un factor de resiliencia favorable para ayudar a la persona que recibe el impacto de una crisis a recuperar el balance previo de funcionamiento 
o a superarlo (Lizardi, 1999; Lizardi, 2000). Esta también ha sido mi experiencia trabajando con otros grupos poblacionales.

La espiritualidad trasciende a la religión y por lo general cuando he trabajado con personas de diversos credos como católicos, evángelicos, budistas y otros, he encontrado que todos promueven un sentido de solidaridad, amor y sana convivencia. Sin embargo, cuando la persona adjudica un valor mayor a la religión que a la fe y la espiritualidad, a veces existen contradicciones entre su discurso y su conducta. En estos casos el/la trabajador/a social debe ayudar a la persona a reflexionar sobre dichos conflictos sin invadir el derecho a la diversidad y a la libertad de credo. Es importante que si el profesional no se siente cómodo/a o preparado/a para intervenir en esta dimensión de la conducta humana, pueda prepararse para hacerlo o buscar asesoramiento. También puede considerarse el coordinar esfuerzos con profesionales específicamente adiestrados en aspectos teológicos y espirituales.

De acuerdo a Greene \& Armenta (citados en Greene \& Cohen, 2005) los trabajadores y trabajadoras sociales que sustentan una perspectiva de resiliencia incluyen en su repertorio de estrategias y técnicas de intervención las siguientes:

- reconocen la pérdida del participante, la vulnerabilidad y las implicaciones para el futuro

- identifican las fuentes de estrés del participante

- reconocen el estrés

- estabilizan o normalizan la situación

- ayudan al participante a tomar el control de la situación

- proveen recursos para el cambio

- promueven la auto-eficacia

- colaboran en los cambios que desea el o la participante

- fortalecen las destrezas de tomar decisiones

- dan énfasis a emociones positivas

- escuchan y validan las historias de los y las participantes

- ayudan a los y las participantes a darle sentido a situaciones críticas 
- ayudan a los y las participantes a visualizar las ganancias de las circunstancias adversas

- ayudan a los y las participantes a trascender la situación inmediata

Las estrategias de intervención basadas en una perspectiva de resiliencia se desarrollan cuando los trabajadores y trabajadoras sociales reconocen que cada persona tiene una serie de fortalezas personales y sociales las cuales ha aprendido y utilizado durante su vida y que pueden reactivarse mediante la intervención profesional de los trabajadores y trabajadoras sociales (Greene \& Cohen, 2005). Éstas han sido incorporadas a la intervención en crisis con diferentes poblaciones como pacientes de cáncer de mama, adultos mayores y víctimas de desastres de la naturaleza (Lizardi, 1999; Greene y \& Cohen, 2005; Molina, 2000). En mi experiencia directa con sobrevivientes de violencia doméstica y de cáncer he podido aplicar la perspectiva de resiliencia con resultados exitosos. Además he recibido el mensaje de que el dolor humano es una característica que nos iguala y nos une a todos y a todas. En el momento de adversidad se tiende a olvidar todas las categorías que los humanos nos trazamos en nuestra existencia. Ante el dolor no hay género, clase social, raza, etnia o cultura. El dolor nos unifica, aunque lo expresemos e interpretemos de diferentes maneras utilizando las categorías previamente descritas. Lo que quiero decir es que la adversidad saca de los humanos capacidades que no pensamos que pudieran estar allí, aunque siempre hubieran estado, esas capacidades que te mueven a sacar las garras como las fieras o a renovar tus plumas como el águila. Ese mensaje lo he podido escuchar de mis participantes ya sean sobrevivientes de cáncer o sobrevivientes de violencia doméstica, cuando estamos en una sesión individual o grupal. La perspectiva de resiliencia es recomendable en la prevención ya sea a nivel primario, cuando aún no se presentan los indicios de un problema, como a nivel secundario, cuando la situación está en crisis o inclusive a nivel terciario con propósitos de tratamiento. 


\section{Principios de intervención profesional}

La intervención está dirigida por los siguientes principios:

- El trabajador ó trabajadora social descarta la posición de persona experta y asume el rol de facilitador/a de procesos de crecimiento y cambio.

- Se descarta la visión de cliente y paciente y se utiliza la visión de participante.

- Las familias participantes tienen la capacidad para aportar al proceso de intervención y a su transformación.

- La intervención fomenta la creación de redes sociales en la familia, la comunidad y la sociedad.

- Las situaciones de las personas participantes de los servicios deben visualizarse dentro de un contexto sociopolítico.

- La mujer sobreviviente de violencia y el hombre agresor necesitan un espacio para cuestionar o retar patrones socio-culturales e ideologías que limiten su desarrollo.

- La intervención da énfasis a las fortalezas y al desarrollo de resiliencia y las limitaciones se visualizan como retos vitales.

- Se fomenta el que el hombre y la mujer desarrollen una identidad que no esté definida por las relaciones que tenga con otras personas sino por sus propios logros o fortalezas.

- Se visualiza a la trabajadora o trabajador social como facilitador/a, educador/a, intercesor/a, investigador/a y promotor/a de cambios a nivel de los sub-sistemas cognoscitivo, afectivo, interpersonal, conductual y espiritual de las/os participantes.

- Se visualiza que la trabajadora o el trabajador social también puede influenciar la política social directa o indirectamente a través de las relaciones colaterales, colegiales o socio- políticas.

- Se visualiza a las personas con capacidades de enfrentamiento o resiliencia ante situaciones de alto estrés, crisis, trauma u otras situaciones de riesgo. 
- A través de la intervención la.persona se capacita y puede ser recurso para efectuar cambios en el contexto ambiental (visión de apoderamiento).

- Toda persona tiene fortalezas.

- La relación profesional está basada en el respeto mutuo.

- La intervención es un ${ }_{l}$ oceso participativo.

- La intervención su visualiza como un proceso de construcción sistémica entre las personas participantes y el/la trabajadora social y propone la desaparición del poder-control unilateral del trabajador/a.

- La trabajadora o trabajador social reconoce sus construcciones sociales y cómo las mismas impactan el proceso de intervención, mediante un constante análisis de las mismas.

- La objetividad absoluta no existe por lo cual el análisis del trabajador/a social a la situación de la participante nunca se puede considerar completamente objetivo.

- La intervención construye una descripción de la realidad de la participante donde ambas son responsables y activas en el proceso de solucionar el problema.

- La violencia es un problema cuyas raíces surgen de estructuras sociales que promueven relaciones de opresión y desigualdad entre los géneros.

- La violencia requiere un entendimiento y análisis dentro de un contexto social abarcador que considere la opresión, los derechos humanos, la justicia social y la construcción de género.

- La intervención profesional debe dirigir sus esfuerzos hacia la intercesión, la prevención y los cambios sociales que complementen los esfuerzos remediales de los problemas sociales. 


\section{Blancos de intervención}

El modelo es aplicable tanto a la víctima o sobreviviente como al agresor o a la agresora considerando las diferentes variables que intervienen en la situación tales como: el momento en que se solicitan los servicios (nivel de prevención), los factores de riesgo y seguridad, la gravedad de la situación, los aspectos legales, las necesidades, intereses, fortalezas y situaciones de vida de la persona y si el servicio es voluntario o la persona es referida como parte de un plan de servicios. Hay que considerar que la intervención nunca debe poner en riesgo la seguridad de la víctima o sobreviviente y tomar las medidas apropiadas para garantizar la integridad física y psicológica de éstas. Cuando existe una orden de protección en una pareja o se considera necesaria para la seguridad de la sobreviviente y es ésta quien solicita los servicios, la agencia no ofrece los servicios al agresor. Cuando un agresor solicita el servicio y existe una orden de protección en su contra, la agencia no ofrece los servicios a la pareja de éste. No obstante, existe la alternativa de conectar a cualquiera de las partes con otros recursos comunitarios o de utilizar facilidades físicas alternas, que garanticen la seguridad de la sobreviviente. Por lo general estas situaciones no son comunes. En la mayoría de los casos de carácter extremo, la relación de pareja se ha roto al momento de solicitar los servicios.

Hay que tomar en cuenta que existen unas diferencias básicas entre el comportamiento de la víctima o sobreviviente y el agresor o agresora. En la gran mayoría de las situaciones la persona agresora es del género masculino y la persona sobreviviente es del género femenino. Hay que considerar que la víctima o sobreviviente es la que recibe el impacto negativo mayor de la violencia. En la mayoría de las ocasiones éstas acuden al Programa en periodos de crisis. Por otro lado, el agresor llega pensando que no ha hecho nada incorrecto, que lo han juzgado mal, o cuando admite que ha cometido el abuso, minimiza el impacto y busca justificaciones como se desprende del siguiente testimonio: "Yo hablo duro pero es que hablo así, ese es mi tono de voz, pero la gente cree que estoy peleando. Cuando pasó "aquello" la social dijo algo como violencia doméstica que yo no 
entiendo, pero yo no le di a mi mujer, bueno reconozco que le di, pero no con violencia doméstica de esa y la social escribió que yo niego la violencia doméstica pero que acepto que le he pegado a mi esposa" (Fernández, 2006).

En nuestra experiencia, el agresor no confronta problemas de autoestima al mismo nivel que la sobreviviente y cuando decide romper con una relación lo hace con más facilidad que ésta. Esto se explica por el hecho de que ha sido socializado en una sociedad patriarcal en la cual se visualiza la violencia y la fuerza como parte del desempeño del hombre. Esto también explica su asombro cuando se le refiere para recibir los servicios de la agencia. Una expresión común es decir: "yo nunca le he puesto una mano encima a mi mujer", aludiendo a su definición de lo que constituye violencia. Todas estas diferencias observadas en el comportamiento y actitudes de las sobrevivientes y los agresores y en el contexto social de donde provienen, tienen que tomarse en cuenta en la intervención.

La intervención con la sobreviviente va dirigida a que ésta gane control sobre su vida, no tolere el abuso y reclame sus derechos; mientras que la intervención con el agresor va encaminada a que éste asuma responsabilidad por sus actos, deje de ejercer un control y poder desmedido y respete a su pareja. Por tales razones aunque conceptual y metodológicamente el modelo provee tanto para la intervención con la víctima o sobreviviente como con el agresor, la intervención es cualitativamente diferente y diseñada tomando en cuenta las diferentes variables de cada situación en particular. La intervención puede llevarse a cabo a nivel individual o a nivel grupal o en ambos niveles. En algunas situaciones se ha intervenido con parejas tomando en cuenta una serie de factores que explicaré más adelante. 


\section{Niveles de intervención}

\section{A. Intervención individual}

El modelo propuesto visualiza la intervención profesional manejando el problema de la violencia doméstica a diferentes niveles de intervención. A nivel del micro-sistema se visualiza la intervención con los actores o actrices inmediatos de este problema social. Aquí se puede intervenir por separado con la sobreviviente, con el agresor o con los hijos de éstos. Se establece claramente que la intervención debe ser por separado por los riesgos que podría representar para la sobreviviente y sus hijos.

La intervención a nivel individual ocurre casi siempre a nivel de prevención secundaria. Este tipo de prevención toma la forma de intervención en crisis, cuando las destrezas de enfrentamiento y solución de problemas colapsan o se encuentran altamente amenazados (Slakieu, 1996; James y Gilliland, 2005). Cabe señalar que se da prioridad a la preservación de la integridad física y psíquica de la sobreviviente de la agresión y de sus hijos.

De ninguna manera se antepone la meta de la preservación familiar a la meta de la preservación de la integridad física o psicológica de las personas que solicitan los servicios. Sin embargo, cuando se trabaja a nivel de prevención, no necesariamente debe descartarse dicho esfuerzo, si no representa un riesgo o amenaza para las personas participantes y si el trabajador o la trabajadora social posee los conocimientos y destrezas necesarios para el manejo de dicha situación.

Por mucho tiempo la intervención con el agresor ha estado relegada a un segundo plano y en manos de otros profesionales de la conducta. Sin embargo, nuestra experiencia profesional nos confirma, que la persona que comete el acto de agresión debe ser un blanco de intervención del trabajador o trabajadora social que tenga un adiestramiento apropiado. Otra de las justificaciones para intervenir con personas que agraden a su pareja es que en muchas 
ocasiones la mujer u hombre víctima o sobreviviente decide permanecer con ésta y no debe ser la posición del profesional imponer mediante amenazas el abandono o rompimiento de la relación. La profesión de trabajo social nos habla del principio de la libre determinación. No podemos convertirnos en figuras opresoras cuando estamos en contra de la opresión. Sin embargo, si tenemos la visión de que las personas participantes de nuestros servicios pueden apoderarse y tienen la capacidad de resiliencia, podemos utilizar estrategias de intervención para que sea esta persona apoderada, capacitada y pensante quien con nuestro apoyo y el de otros sistemas sociales, rompa con la situación violenta. Es decir, romper el ciclo de la violencia no siempre se logra escapando, sino cambiando la conducta violenta de la persona agresora, la cual no es violenta en otras relaciones sociales, sin embargo, es violenta con su pareja.

Una razón adicional para intervenir con la persona que arremete en una relación de pareja es que es muy frecuente, en Puerto Rico, que dichas personas terminen una relación y se involucren en una relación nueva en un corto periodo de tiempo. Esta situación es frecuente tanto para la persona sobreviviente como para el agresor o agresora. Por tal razón, la intervención podría incluir a la persona agresora, una vez se haga la ponderación y análisis de la situación y los niveles de mortalidad o letalidad, que indican cuán expuesta o vulnerable está la persona de cometer un acto de daño físico hacia sí misma o hacia otra persona, no estén altos. Para esto se han elaborado criterios e instrumentos específicos y se han adoptado algunos de otros autores (James y Gilliland, 2005). Estos indicadores ayudan a determinar el tipo de intervención a ofrecerse tanto a la víctima o sobreviviente como a la persona agresora.

Ya sea que se trabaje con la sobreviviente o con el agresor, la intervención en el nivel de prevención secundaria tiene como propósito el que la persona reestablezca por lo menos su nivel previo de funcionamiento, pueda conectarse con recursos familiares o comunitarios y se mantenga con los niveles de mortalidad bajos. La intervención en crisis manejada en un periodo 
de cuatro a seis semanas de ocurrir el evento precipitante previene el que la persona adopte patrones maladaptativos y caiga en un deterioro psicológico (Slakieu, 1996; James y Gilliland, 2005).Además en el periodo de la crisis activa la persona está más receptiva al cambio y se puede trabajar con las capacidades de resiliencia (Lizardi, 1999).

\section{B. Intervención con familias}

A nivel familiar se interviene con las mujeres sobrevivientes y sus hijos. Por lo general, estas familias han superado una crisis familiar, han roto con el ciclo de la violencia, desean fortalecer sus capacidades de resiliencia y desarrollarse académicamente, adquirir una vivienda propia, conseguir un empleo o desarrollar su propia empresa. La agencia ha desarrollado acuerdos de colaboración con diferentes organizaciones comunitarias con las cuales se coordinan los servicios de educación, empleo, vivienda y proyectos de auto-gestión. Además se trabaja con el concepto de apoderamiento y autogestión motivando a las participantes a participar en este tipo de programas. El rol fundamental de la intervención a este nivel es procurar facilitar que la participante recupere el control sobre su vida a través del desarrollo de sus fortalezas. Aquí la intervención también focaliza en las perspectivas de fortalezas y resiliencia (Long, Tice \& Morrison, 2006; Fraser, 2004; Walsh, 2006).

La intervención con parejas que han experimentado violencia de tipo psicológico es una modalidad que se encuentra bajo estudio. La mayor parte de los profesionales mira este tipo de intervención con cierto recelo por las posibles implicaciones a la seguridad. No obstante, debemos recordar que uno de los principios fundamentales del trabajo social es la libre determinación. En el trabajo directo con sobrevivientes de violencia doméstica y con agresores no hemos encontrado dos situaciones que sean exactamente iguales. La investigación e intervención con este grupo poblacional ha contribuido a ampliar nuestra percepción del problema y a descartar los estereotipos y falacias que se diseminan sobre estas personas. 
Aunque existen unas características en común en el problema de la violencia doméstica la manifestación específica del mismo es tan diversa como los actores y actrices que la protagonizan. Volvemos a afirmar que aunque la preservación de la familia no es la meta principal que dirige nuestra intervención, no descartamos que en algunas situaciones sea posible llevarla a cabo por profesionales bien adiestrados en la intervención con familias y la intervención en crisis.

Para considerar el trabajar con situaciones de violencia doméstica de tipo psicológico a nivel de pareja debe darse una serie de requisitos entre los cuales se encuentran los siguientes:

- que haya habido una intervención a nivel individual (por separado) con ambas partes del problema.

- que no se haya expedido una orden de protección

- que se haya hecho un análisis de los niveles de mortalidad y la seguridad de las partes no esté afectada o amenazada

- que ambas partes hayan expresado el interés de terminar con la situación de violencia y de trabajar a nivel de pareja

- que no haya habido agresiones de tipo físico

- que ambas partes se comprometan con el cambio

- que no se trate de un problema crónico

- que no haya un patrón de violencia generalizada en otros contextos

- si ocurriese alguna agresión física se descontinuaría el tipo de intervención y se tomarían otras medidas

Este tipo de intervención tiene un enfoque de prevención y merece se evalúe como una alternativa adicional muy pocas veces considerada. 


\section{Intervención con grupos}

La intervención grupal es la modalidad que más énfasis ha tenido por lo exitoso de los resultados. El Programa comenzó trabajando con la sobreviviente de violencia doméstica bajo los auspicios del Departamento de Justicia y del Departamento de la Familia y dicha experiencia contribuyó a desarrollar estrategias de intervención con los hombres y mujeres agresores/as bajo el auspicio del Departamento de la Familia. La investigación e intervención con ambas poblaciones llama la atención al hecho de que la violencia doméstica es un problema social, muy complejo y multidimensional, por lo cual las alternativas de intervención deben tener un enfoque cónsono con el mismo. La intervención grupal con la mujer sobreviviente se desarrolla a través de grupos denominados de apoyo los cuales son de naturaleza socioeducativa.

Con el hombre agresor y la mujer agresora la intervención grupal se lleva a cabo a través de grupos denominados de re-socialización masculina o femenina, los cuales también son de naturaleza socioeducativa. Los grupos para agresores o para sobrevivientes constan de diez sesiones de dos horas de duración cada sesión. La intervención grupal se considera como alternativa idónea para programas con enfoques de prevención a todos los niveles (Lizardi, 2002; Lizardi \& Molina, 2003; Lizardi \& Molina, 2005; Fernández \& Molina,2005; Molina, 2006).

\section{Intervención con la comunidad}

Las estrategias de intervención a nivel macro que se han llevado a cabo a través del personal del Programa AFANA se han realizado a través de participación en talleres de educación a la comunidad, a través de exposición en los medios de comunicación masiva, a través de publicaciones profesionales, ponencias y participación en actividades profesionales, consultoría, investigaciones, adiestramientos y asesoramiento a personas que implantan la política social. Los talleres educativos giran en torno a diferentes 
temas tales como: la prevención de la violencia, desarrollo de programas, estrategias de intervención y modalidades de evaluación de programas. De esta manera se amplían los niveles de intervención y se ha contribuido en cierto grado a impactar la política social relacionada a la prevención e intervención con la violencia doméstica.

\section{Metas de la intervención profesional}

La intervención profesional de los trabajadores y trabajadoras sociales va dirigida a las siguientes metas:

- Preservar la integridad física y emocional de las personas participantes de los servicios.

- Trabajar con los efectos psico-sociales de haber vivido en una relación de opresión y violencia.

- Desarrollar asertividad para sustituir patrones pasivos, agresivos o manipuladores de comunicación e interacción en las relaciones sociales de las personas participantes.

- Ampliar la visión de los roles de género y promover la búsqueda de relaciones de pareja matizadas por la igualdad y la justicia.

- De-construcción de ideas que promuevan la opresión.

- Conectar a las personas participantes con redes de apoyo formales (agencias de servicios, grupos de acción social, etc.).

- Conectar a las personas participantes de los servicios con redes de apoyo informales (familiares, vecinos, amigos, etc.)

- Crear conciencia sobre el impacto físico, social y emocional de la violencia.

- Romper con el ciclo de violencia en las relaciones de pareja.

- Desarrollar destrezas sociales para la toma de decisiones y el manejo de sentimientos.

- Desarrollar auto-percepciones saludables. 
- Retar ideas o discursos que contribuyan a fomentar o a justificar la violencia.

- Conocer la Ley 54 del 15 de agosto de 1989.

- Liberarse de patrones socio-culturales e ideologías que limitan el desarrollo y las relaciones de los seres humanos.

- Establecer acuerdos de colaboración con diferentes recursos de la comunidad.

- Llevar a cabo actividades educativas de concienciación y prevención de la violencia.

Aunque existen elementos comunes y complementarios en las construcciones sociales que presenta el hombre o la mujer agresor/ a y la mujer sobreviviente de violencia doméstica, hay que tener en cuenta que el/la agresor/a es la persona que viola la ley y comete la conducta transgresora mientras que la mujer sobreviviente es la que recibe el impacto negativo del delito. El agresor tiene que reconocer su responsabilidad en el problema y comprometerse a eliminar la conducta maltratante. Por esta razón el primer paso es ayudar a crear conciencia sobre el problema y las implicaciones físicas, familiares, legales, sociales y económicas del mismo. Aquí visualizamos al agresor o agresora en un entorno social paradójico que promueve y construye el problema por un lado y por el otro sanciona y penaliza el mismo.

El que un agresor o agresora acepte su responsabilidad y se conciencie de las implicaciones de la conducta maltratante para él/ella y toda la familia es un logro fundamental sin el cual sería imposible lograr otros cambios. Por eso es que hasta cierto punto la intervención con el agresor es más directiva que con la sobreviviente. Las mujeres agresoras son menos y participan en grupos de resocialización femenina. Por el momento se han separado los grupos por género, para garantizar mayor estabilidad en el grupo, aunque no se descarta la posibilidad de tener grupos de agresores y agresoras en un mismo grupo en un futuro. 
Mientras que con la sobreviviente de violencia se refuerzan las respuestas de enfrentamiento y resiliencia, la intervención con el/ la agresor/a va dirigida a que descontinúe la conducta maltratante y a que incorpore nuevas formas de solucionar problemas y manejar sentimientos. Sin embargo, en mi opinión la perspectiva de resiliencia también puede ser un marco conceptual que guíe la intervención con la persona agresora y en estos momentos se está considerando en la intervención. Cabe mencionar que los grupos de re-socialización masculina son dirigidos por una pareja de trabajadores sociales, una mujer y un hombre. Al hacer esto se facilita el trabajo con la de-construcción de los roles de género rígidos que promueven respuestas violentas. Con esto se presenta un modelo de figura femenina en una relación de poder compartido de igualdad y respeto. De esta manera se promueve el manejo de conflictos en una situación grupal protegida.

La figura del profesional de trabajo social masculino también provee de un modelo donde el hombre puede aceptar que una mujer tenga un papel protagónico de mayor fuerza. El trabajador social desempeña un rol socio-emocional en el grupo, se sienta junto a los participantes y está atento a todos sus comentarios, los cuales refuerza y presenta para discusión y reto. La trabajadora social asume un rol más directivo en la discusión del contenido educativo del grupo. En mis participaciones con este grupo y con algunos de sus integrantes he podido visualizar que la diferenciación de los roles de los facilitadores y el género de éstos se convierte en un factor positivo para el cambio y el logro de las metas del grupo. También he podido visualizar que los participantes identifican al trabajador social como una figura de confianza y empatía y a la trabajadora social la identifican como a una líder instrumental. Esta relación de los roles y el género representa un reto a la ideología dominante de la sociedad patriarcal que coloca al hombre en roles mayormente instrumentales y a la mujer en roles donde se da mayor énfasis a los aspectos socio-emocionales. Simbólicamente de esta manera ya se presenta un reto a visiones rígidas de roles de género. La posición y roles de los facilitadores no es rígida y da lugar a un intercambio de posiciones. En ocasiones la trabajadora social que está a cargo del grupo es sustituida por otra 
trabajadora social que cambia completamente el estilo de participación proveyendo el que los participantes açepten la diversidad y puedan aplicar este aprendizaje a sus situaciones de vida.

Otro de los aspectos significativos en el grupo de re-socialización masculina y femenina es la de-construcción de la conducta maltratante. Esto no solamente se logra a través del contenido grupal sino a través del mismo proceso grupal. Este proceso es similar al que ocurre en el grupo de mujeres sobrevivientes cuando se trabaja con las ideas y discursos que recogen la construcción social de la violencia. Los grupos son participativos para promover que a través del diálogo se pueda trabajar con las construcciones sociales de los miembros.

\section{Técnicas de intervención}

Para trabajar con la de-construcción de la violencia en la sobreviviente y el agresor se invita a éstos a reflexionar sobre sus ideas de lo que significa una relación de pareja, sobre los roles de género y sobre las implicaciones de la violencia, todo lo cual proviene del proceso de socialización y refleja la ideología dominante de una sociedad machista y patriarcal. Se reflexiona sobre sus ideas, sueños, aspiraciones y metas. Tanto con la sobreviviente como con el agresor, se trabaja con ideas que contribuyen a que existan relaciones violentas. En este modelo adoptamos la perspectiva del construccionismo social (Gergen, 1994; Bravo Urzúa, 2002; Rozo, 2002; Gergen, McNamee \&Barret, 2001). Una de las técnicas que Gergen, MacNamee \& Barret (2001) proponen para lograr cambios en las relaciones de las personas es el diálogo transformador. Este diálogo es: cualquier forma de intercambio verbal que pueda transformar una relación entre dos partes comprometidas con realidades y prácticas separadas y antagónicas. A través de esta técnica se invita a las partes a visualizar la relación en lugar de hacer acusaciones y culparse una a la otra. Además se da énfasis en el diálogo a hablar 
en términos personales en vez de referirse a conceptos o principios abstractos o generales con los cuales los/as participantes no pueden relacionarse y a los cuales no le encuentran ningún significado. E1 evitar este tipo de comunicación reduce al máximo la resistencia. Así cuando una persona que ha cometido un acto violento te dice: "reconozco que le he pegado a mi esposa, pero no soy un agresor” (Fernández, 2006) sería fútil que el/la trabajador/a social invirtiera los esfuerzos en cambiar la autodefinición de éste; por el contrario, el diálogo transformador provee la oportunidad para que esta persona cuente su historia y exprese la interpretación que le da a la misma desde su realidad subjetiva. En este caso es de mayor importancia que la persona asuma responsabilidad por su conducta, que analice las consecuencias de la misma y que pueda visualizar cómo ésta afecta a las demás personas.

También se trata de que la persona analice su comportamiento desde una perspectiva colectiva como lo es reflexionar sobre el proceso de socialización por género. En resumen, el diálogo transformador en la intervención pone especial interés en la responsabilidad de la persona participante por las relaciones, la expresión sobre sí misma, la reflexión y la creación mutua de nuevas realidades (Gergen, MacNamee \& Barret, 2001). Como parte de las estrategias de intervención tanto a nivel individual como grupal se trabaja con las construcciones sociales de las sobrevivientes y de los agresores o agresoras. Se lleva a éstos/as a conversar y reflexionar sobre sus discursos, lo que representan dichos discursos y la emoción y conducta que viene como resultado de los mismos. Es interesante el hecho que no necesariamente tiene que ser el o la facilitadora de los grupos quien rete dichas ideas, expresadas en forma de discurso, sino que los miembros del grupo asumen una posición activa en dicho proceso. Lo importante es que no se impone una visión, sino que se lleva a analizar o a reflexionar sobre otras ideas que sirven de realidad alterna. 
A continuación se presentan ejemplos de discursos sobre los cuales se invita a las sobrevivientes a reflexionar:

- “Él me pega pero no es mujeriego, ni bebe. (Minimizar o justificar las conductas violentas.)

- “Él solamente me tiene a mí". (Pensar que debe "salvar" o es responsable de que el agresor" busque ayuda.")

- “Es verdad que él me agredió, pero yo tuve la culpa por pelearle porque llegó tarde." (Pensar que la sobreviviente es responsable de la conducta del agresor.)

- "No sé que voy a hacer ahora, pues él siempre se encargaba de todo." (Pensar que no puede funcionar sin su pareja.)

- "Los hombres son más inteligentes que las mujeres." (Estereotipos de género.)

- “Los hombres son unos puercos.”(Estereotipos de género.)

- “Que sea lo que Dios quiera.” (Percibirse a sí mismo o a sí misma como incapaz de romper con el ciclo de la violencia.)

La reunión grupal provee el contexto para invitar a los/as participantes a considerar discursos alternativos. Este tipo de análisis de las narraciones ha resultado beneficioso tanto con la sobreviviente como con el agresor, ya que en muchas ocasiones ambos sostienen ideas parecidas en relación al género, a la violencia y a las relaciones de pareja (Fernández, 2006). No obstante, coincido con Rozo (2002) en el sentido de que esa narración debe traducirse en acciones nuevas para enfrentar antiguas relaciones de manera que propicie cambios en el sistema y éste pueda superar la situación considerada antes como problema. Cabe señalar que aunque los énfasis y los roles de los facilitadores grupales son diferentes, tanto los grupos de sobrevivientes como los grupos de los agresores/as siguen una temática similar. En ocasiones, cuando la ponderación de la situación lo indica, se trabaja con el hombre a nivel del grupo de re-socialización masculina y con la mujer sobreviviente en el grupo de apoyo. Esto ha resultado beneficioso pues ayuda a los participantes a romper con el ciclo de la violencia por el cese de la misma o por la ruptura 
del vínculo matrimonial. No se impone ninguna de estas dos alternativas pues se respeta la libre determinación de los participantes de los servicios. Como se explicó anteriormente si existe una orden de protección este tipo de intervención no sería apropiado y se buscarían modos alternos de ofrecer los servicios.

Otras técnicas de intervención con grupos de sobrevivientes y grupos de agresores incluyen lo siguiente:

- Discusión de las implicaciones físicas, emocionales, sociales, económicas y legales de la violencia. Aquí se discute la Ley 54 de 15 de agosto de 1989. Cabe señalar que en el grupo de los hombres se ofrece énfasis a las penalidades y medidas punitivas con el propósito de que éstos se conciencien de los posibles efectos de su conducta. Por otro lado en los grupos de sobrevivientes se da énfasis a la discusión de las órdenes de protección y las medidas civiles y criminales que establece la ley.

- Discusión sobre diversos tópicos tales como: relaciones de pareja, toma de decisiones, solución de problemas, comunicación efectiva, formación de género y autoestima entre otros.

- Diario reflexivo. Este tipo de técnica se ha encontrado que es más útil con mujeres que con hombres ya sea por el nivel educativo o por el contexto de socialización. Por lo general a los hombres que reciben los servicios se les hace más difícil escribir que a las mujeres.

- Análisis de lecturas reflexivas. En este tipo de técnica no hemos encontrado mucha diferencia entre los géneros. La aplicabilidad está más relacionada al trasfondo sociocultural que al género. La experiencia ha sido que la técnica genera mucha discusión entre los hombres y entre las mujeres.

- Uso de narraciones personales. Esta técnica es la preferida por los agresores y por las sobrevivientes. La narración personal en ambos grupos se antepone a cualquier otra técnica de intervención a nivel individual o grupal. 
- Role playing o dramatizaciones de roles para ilustrar conductas y situaciones sociales.

- Testimonios de otras participantes. Esta técnica ha sido utilizada solamente con las sobrevivientes, pero no se descarta su utilización al trabajar con agresores o agresoras. La ventaja es poder observar a una persona que en situaciones similares pudo superar la situación.

- Películas y videos. Estas técnicas son de gran efectividad en ambos grupos. Se recomienda evaluar las mismas antes de utilizarlas y desarrollar una guía de preguntas para su uso.

- Música popular, teatro popular, refranes y la reflexión sobre los mensajes que llevan.

- Humor como estrategia de lidiar con situaciones de alto estrés y para explicar situaciones sociales.

\section{Evaluación}

La evaluación del programa se hace utilizando diversas estrategias de investigación cuantitativa y cualitativa para medir cambios como resultados de la intervención sobre las siguientes variables:

- Nivel de autoestima

- Conocimientos y actitudes sobre la violencia

- Conducta violenta y no violenta

- Manejo de estrés

- Comunicación asertiva

- Incidentes de violencia en el hogar

- Satisfacción con los servicios

- Contenido, metodología y recursos de los grupos

- Número de personas atendidas 


\section{Conclusiones y recomendaciones}

La violencia doméstica es un problema complejo y multidimensional. El presente trabajo no pretende ser una muestra exhaustiva de todas las posibilidades que existen en la prevención e intervención con la violencia doméstica. Sin embargo, puede concluirse que los trabajadores y trabajadoras sociales necesitamos revisar constantemente nuestro acervo de estrategias de intervención y prepararnos para presentar alternativas de solución a este problema donde no se minimice la capacidad de cambio y de tomar decisiones de los/as participantes de los servicios.

- Re-enfocar la política social para que haya más acercamientos o estrategias de prevención primaria en escuelas, universidades, hospitales, centros de salud mental, tribunales, iglesias, etcétera. El esfuerzo debe ser compartido por los diversos sectores: agencias gubernamentales, organizaciones de base comunitaria, instituciones de educación superior, iglesias etc. Dichos esfuerzos deben ser sistemáticos y continuos. Esto quiere decir que partan de un currículo establecido y estén fundamentados en resultados de investigaciones sociales. Además se deben contar con un plan para evaluar su efectividad.

- Seguir apoyando y ampliando esfuerzos programáticos dirigidos a lograr la autosuficiencia económica de mujeres sobrevivientes de violencia doméstica.

- Revisar continuamente los currículos de las instituciones educativas del país de manera que los niños y niñas puedan recibir una educación que se aparte de ideologías que promuevan relaciones de inequidad o visiones rígidas de relaciones o roles de género.

- Procurar de tener una mayor aportación de los medios de comunicación masiva hacia llevar un mensaje que promueva relaciones de justicia y paz, que no denigren la figura de la mujer o menosprecien la aportación de éstas a la sociedad. 
- Requerir que las parejas que consideren legalizar una unión matrimonial pasen por la experiencia de talleres educativos de prevención de la violencia, libres de costo.

- Incluir en los currículos de las escuelas a nivel primario, intermedio y superior, material educativo sobre lo que es la violencia y sus implicaciones en la vida familiar. La educación debe preparar no solamente en los aspectos de conocimientos y destrezas, sino en los aspectos afectivos que incluya la reflexión y el análisis de discursos y el diálogo transformador. Esta práctica no debe limitarse y debe extenderse a las instituciones de educación universitaria especialmente en los currículos de las escuelas y programas de trabajo social en los diferentes niveles educativos.

- Fortalecer en los currículos de las escuelas o programas de trabajo social contenido que ayude a los estudiantes a desarrollar los conocimientos, destrezas y actitudes necesarias para trabajar con la prevención e intervención con situaciones de violencia doméstica .Especial énfasis debe ofrecerse al aspecto de las actitudes y a los aspectos éticos al intervenir con estas poblaciones.

- Ofrecer mayor énfasis a la perspectiva de fortalezas y al desarrollo de la resiliencia a todos los niveles de prevención.

- Fomentar estudios que arrojen luz sobre las capacidades de resiliencia de manera que este conocimiento pueda incorporarse en programas de prevención, en el análisis de la política social y en los currículos de las escuelas y programas de trabajo social.

- Evaluar la efectividad de los programas de servicios existentes para intervenir y prevenir la violencia doméstica especialmente a aquellos subvencionados por fondos gubernamentales.

- Que se apoyen iniciativas de prevención y de servicios para el hombre y la mujer que presentan una conducta violenta hacia su pareja. La experiencia nos afirma que existe dicha necesidad. En la actualidad hay programas de desvío para 
agresores y muy pocas alternativas de servicios para situaciones a nivel de crisis o programas de carácter voluntario que no tienen presión legal como lo es el Proyecto de Re-socialización Masculina y Femenina del Instituto IGODI, Programa de Apoyo Familiar a Niños y Adultos de Gurabo.

- Establecer o fortalecer los Programas de Trabajo Social Ocupacional o de Trabajo Social Obrero para personas cuyo escenario de trabajo les hace vulnerables a experimentar violencia en su relación de pareja como lo son las personas que trabajan en la policía o escenarios similares, donde poseen armas de fuego. Dichas personas continuamente enfrentan situaciones de alto estrés, factor que aumenta su vulnerabilidad y riesgo y pueden precipitar crisis circunstanciales.

- Fortalecer los Programas de Trabajo Social Ocupacional en los escenarios de trabajo para que implanten medidas de prevención primaria con los empleados ya que existen factores sociales, económicos y políticos que inciden en la vida familiar y en la relación de pareja y pueden desencadenar o precipitar situaciones de violencia familiar. Los Programas de Trabajo Social Ocupacional pueden ser auspiciados por las agencias que les emplean o por las uniones de empleados. Los esfuerzos preventivos con estos empleados no deben ser esporádicos sino sistemáticos y con un enfoque de trabajo social que visualice a la persona en su entorno ya sea en el empleo, en la familia y en la comunidad.

- Que los trabajadores y trabajadoras sociales incursionemos en el campo de Trabajo Social Ocupacional que se ha dejado un poco olvidado y es tan importante, como lo hizo el compañero José Joaquín Parrilla el cual no está físicamente con nosotros, pero nos dejó su legado.

- Fortalecer los Programas y Escuelas de Trabajo Social sus currículos para que se ofrezca mayor énfasis al trabajo social ocupacional. 
En estos momentos recuerdo una reflexión cuyo autor/a desconozco pero que establecía que: "el éxito comienza con la voluntad". Quisiera dejarlos/as con este pensamiento para que unamos las voluntades individuales y construyamos un colectivo que aúne esfuerzos y que nunca se rinda, hasta que podamos controlar o eliminar el problema de la violencia doméstica que nos está deteriorando como sociedad.

\section{Referencias}

Bravo Urzúa, J. A. (2002). Hacia una comprensión del construccionismo social de Kenneth Gergen: Recuperado de Internet el 24 de octubre de 2006 de http://members.fortunecity.es/matiassunp// gergen.1 construcc.htm/.

Díaz-Santana, J., García García, D., Guzmán Viera, B., Suárez Murphy, C. (2001). Violencia Doméstica: Transmisión Intergeneracional en Agresores. Tesis de maestría no publicada, Universidad de Puerto Rico.

Fernández, A. (2006). Reconozco que le he pegado a mi esposa, pero no soy un agresor: La otra cara de la violencia doméstica. Manuscrito no publicado.

Fernández, A. \& Molina G.M.(2005, 11 de agosto) Perfil del agresor de violencia doméstica. Recuperado el 4 de octubre de 2006 de http://www.tribunalpr.org/Miscel/Conferencia/pdf/17 ponencia-agresores-v.d.pdf

Fraser, M. (1997). Risk and resilience in childhood. Washington, DC: NASW Press. 
Fong, R. (2002). Empowering multicultural clients by using cultural value and biculturalization of interviews. In M. O'Melia \& K. Miley (Eds.), Pathways to power: Readings in contextual social work practice. Boston: Allyn \& Bacon.

Gergen, K. J. (1994). Realities and relationships. Cambridge: Harvard University Press.

Gergen, K. J.; Mac Namee, S; \& Barret, F.(2001). Towards a vocabulary of transformative dialogue. International Journal of Public Administration, 24, 697-707. Recuperado el 24 de octubre de 2006 de http://www.swarthmore.edu/socsci/ Kgergen/web/page.

Germain C. \& Gitterman, A. (1996).Segunda edición. The life model of social work practice. New York: Columbia University Press.

Greene, R. R. \& Conrad, A. P. (2002). En: Greene, R. R. (2002). Resiliency: An integrated approach to practice, policy, and research. Ed. Washington, DC: NASW Press.

Greene, R. R. \& Cohen, H. L. (2005). Social work with older adults: Changing practice paradigms. Families in society: Journal of contemporary social services, 86(3), 367-373.

James, R. K. \& Gilliland, B. E. (2005). Crisis intervention strategies. Belmont, CA: Thomson:Brooks/Cole.

Lizardi, M. M. (1999). Siempre estaré a tu lado: Una nueva visión ante el cáncer de seno. San Juan, P.R.: Autora. 
Lizardi, M. M. (2000). Niveles de estrés y patrones de adaptación de mujeres diagnosticadas con cáncer de mama del área metropolitana de San Juan, a mayo de 1998. Análisis (2):1, 51-74.

Lizardi, M. M. (2002).Violencia doméstica: Un modelo grupal de intervención. Análisis (3):1, 47-59.

Lizardi, M. M. \& Molina, G.M. (2002, 1 de abril).Mujeres jefas de familia y mujeres sobrevivientes de violencia doméstica: Estrategias de intervención. Ponencia presentada en la Universidad Interamericana de Puerto Rico, Recinto Metropolitano.

Lizardi, M. M. \& Molina, G.M. (2003). La intervención grupal como estrategia de cambio en situaciones de violencia familiar. Ponencia presentada en la tercera Conferencia de Trabajo Social Forense, auspiciada por la Oficina de Servicios Sociales de la Rama judicial de Puerto Rico.

Long, D. D., Tice, C.J. \& Morrison, J. D. (2006). Macro social work practice: A strengths perspective. Belmont, CA: Thomson: Brooks/Cole.

Molina, G. M. (2000). Crisis: Cómo se interviene. San Juan, PR: Editorial Edil.

Norman E. (2000).Ed. Resiliency enhancement: Putting the strengths perspective into social work practice. New York: Columbia University Press. 
Poulin, J. (2005). Strengths based generalist practice: A collaborative Approach. Belmont, CA: Thomson: Brooks/Cole.

Pransky, J. (2003).Prevention from the inside-out. Springfield: Burrell Foundation \& Paradigm Press.

Pransky J. (1991).Prevention: The critical need. Springfield: Burrell Foundation \& Paradigm Press.

Rozo, J. A. (2002). La terapia desde el punto de vista del construccionismo social: ¿Tiene algún sentido? Deparamento de Psicología Experimental, Universidad de Sevilla-España. Recuperado el 24 de octubre de 2006 de http://www.psicología científica.com

Saleebey, D. (2002). The strengths perspective in social work practice. ( $3^{\text {rd }}$ ed.).Boston: Allyn and Bacon. 
